\title{
Delayed Convulsions and Brief Contralateral Hemiparesis After Retrobulbar Block
}

\author{
Elien Pragt, M.D., André A.J. van Zundert, M.D., Ph.D., F.R.C.A., \\ and Chandra M. Kumar, M.B.B.S., M.Sc., F.F.A.R.C.S.I., F.R.C.A.
}

Objective: This case report describes convulsions and hemiparesis after retrobulbar injection with good outcome in a patient undergoing outpatient cryocoagulation of his right eye.

Case Report: We report a young man in which localized convulsions of the ipsilateral face occurred 9 minutes after retrobulbar injection followed shortly by convulsions of the contralateral arm and leg. After the convulsions, the patient experienced left-sided hemiparesis resolving approximately 1 hour after the injection. There was no hemodynamic instability during this period. It was difficult to determine the exact cause of convulsions and hemiparesis.

Conclusions: We believe these complications occurred because of unintentional injection of local anesthetic agent into the subarachnoid space without affecting the brainstem. Possible mechanisms of spread of local anesthetic agent into the central nervous system after retrobulbar block are discussed. Reg Anesth Pain Med 2006; 31:275-278.

Key Words: Retrobulbar block, Complications, Subarachnoid injection, Convulsions, Hemiparesis.

$\mathbf{L}$ ocal anesthesia for major ophthalmic surgical procedures includes peribulbar, retrobulbar, and sub-Tenon's block, ${ }^{1-3}$ but the exact frequency of their use is not known. Although the classical retrobulbar technique ${ }^{4}$ is still in use, the technique has evolved over the years. In current or modern retrobulbar block, a needle $\leq 32 \mathrm{~mm}$ long is inserted through the extreme inferolateral quadrant just below the lateral rectus muscle. ${ }^{5}$ Despite its careful use, sight and/or life-threatening complications have occurred. ${ }^{6-8}$ Numerous case reports of apnea, respiratory difficulties, cardiovascular collapse, convulsions, and paralysis of 1 or more limbs have been reported after local anesthetic spread to unusual neurologic locations. ${ }^{9}$ The exact mechanism of spread is not fully

From the Department of Anesthesiology, ICU, \& Pain Therapy, Catharina Hospital, Brabant Medical School, Eindhoven, The Netherlands (E.P., A.A.J.v.Z.); and Department of Anaesthesia, The James Cook University Hospital, Middlesbrough, United Kingdom.

Accepted for publication January 30, 2006.

Reprint requests: André A.J. van Zundert, M.D., Ph.D., F.R.C.A., Department of Anesthesiology, ICU \& Pain Therapy, Catharina Hospital, Brabant Medical School, Michelangelolaan 2, NL-5623 EJ Eindhoven, The Netherlands. E-mail: zundert@ iae.nl

() 2006 by the American Society of Regional Anesthesia and Pain Medicine.

1098-7339/06/3103-0016\$32.00/0

doi:10.1016/j.rapm.2006.01.004 understood in most cases. A variety of signs and symptoms after injection of local anesthetic agent into the optic nerve sheath have been reported. ${ }^{9}$ A case of contralateral hemiparesis was reported after retrobulbar block in addition to which the patient developed bilateral ophthalmoplegia and loss of consciousness for approximately 2 hours. ${ }^{10}$

We present a case in which localized ipsilateral convulsions occurred 9 minutes after retrobulbar injection, and this was followed shortly by convulsions in the contralateral arm and leg. Contralateral hemiparesis occurred, but the patient recovered full muscle power within an hour. To our knowledge, delayed convulsions and contralateral hemiparesis after retrobulbar block have not been reported.

\section{Case Report}

A 36-year-old man (height $1.73 \mathrm{~m}$, weight $80 \mathrm{~kg}$, and American Society of Anesthesiologists 2) was scheduled for outpatient cryocoagulation of his right eye. The medical history included congenital blindness in the right eye and non-Hodgkin's lymphoma with leptomeningeal metastasis. He received intrathecal chemotherapy and skull radiotherapy more than 5 years before. Although his medical conditions remained stable, he developed radiation retinitis in both eyes. He was addicted to nicotine and 
other contraband drugs. He did not take any other medications. There was no past history of epilepsy.

He received 3 successive laser therapies for retinitis in both eyes under retrobulbar anesthesia without problems. He was sedated with 60 to $80 \mathrm{mg}$ propofol on each occasion. The axial length of each eye was $21.3 \mathrm{~mm}$. On this occasion, he again received $80 \mathrm{mg}$ propofol in divided doses for sedation before instituting the block. The patient was monitored with an electrocardiogram, peripheral oxygen saturation, and noninvasive blood pressure monitoring, and $4 \mathrm{~L} / \mathrm{min}$ oxygen was administered. Oxybuprocaine $0.4 \%$ eye drops were instilled. A retrobulbar technique, similar to previously used technique, was performed in the right eye by the same experienced anesthesiologist. An Atkinson needle, $25-\mathrm{G}, 0.6 \times 32 \mathrm{~mm}$, was introduced through the conjunctiva in the extreme inferotemporal quadrant, and $5 \mathrm{~mL} 1 \%$ ropivacaine was injected slowly after negative aspiration for blood and cerebrospinal fluid. There was no resistance to injection, and the feel of the injection appeared as usual. There was no chemosis or conjunctival hemorrhage. The retrobulbar block was the only block administered, and the facial nerve was not infiltrated. An occulocompression device (Honan balloon) was applied by using a pressure of $25 \mathrm{~mm} \mathrm{Hg}$ to disperse the local anesthetic agent and to reduce the intraocular pressure. The patient was then transferred from the regional anesthesia block area to the operating room.

Nine minutes after the retrobulbar injection, the patient developed a localized twitching on the ipsilateral face followed, within a minute, by convulsions of left arm and leg. The saturation of the patient decreased to $85 \%$ as a consequence of an obstructed airway. A chin lift and administration of $100 \%$ oxygen via face mask resolved the problem. The saturation rapidly increased to $100 \%$ in 1 minute. Diazepam $5 \mathrm{mg}$ was administered intravenously, which stopped convulsions immediately. Cardiovascular and respiratory systems remained stable. No assisted ventilation or vasopressor drugs were required. Surgery was postponed, and the patient was transferred to the recovery room for further observations. The patient slowly recovered from the residual effects of sedatives and could communicate sensibly 20 minutes after the retrobulbar injection. Although a complete neurologic examination by us would have been very valuable, we failed to notice any neurologic deficit because of rapid change in the clinical conditions of the patient. However, subsequently an examination by a neurologist revealed right-sided facial paralysis and left-sided hemiparesis. At this stage, the eye was checked for the residual effects of retrobulbar block, and there were no movements except minor flickers. The patient recovered from the residual effects of retrobulbar injection and sedation after 1 hour and did not recall the event. Full neurologic examination revealed full recovery from muscle weaknesses. Magnetic resonance imaging, an electroencephalogram, and cerebral spinal fluid analysis revealed no signs of cerebral pathology or recurrence of non-Hodgkin's lymphoma.

\section{Discussion}

Our case exhibited 2 separate but brief phenomena: delayed convulsions and temporary hemiparesis. Despite reviewing the literature, we have not been able to understand the mechanism of local anesthetic spread. Several possible mechanisms might account for these events.

Convulsions can occur because of systemic toxicity either because of absorption or intravascular injection ${ }^{9,11}$ and have been reported after both retrobulbar and peribulbar blocks. ${ }^{12,13}$ It is unlikely for late convulsions to occur because of absorption of a local anesthetic agent, especially when a small dose of local anesthetic agent (50 mg ropivacaine) was used for retrobulbar block when used for any other forms of local anesthetic technique. Another possible mechanism is that of unintentional intra-arterial injection and retrograde flow of local anesthetic into the carotid circulation. ${ }^{14}$ This mechanism has been suggested in cases in which immediate grand mal seizure activity has been observed during retrobulbar injection. ${ }^{15}$ If unintentional intra-arterial injection was the probable mechanism in our case, convulsions should have occurred within seconds rather than minutes.

Stress or psychological states could also lead to convulsions in susceptible patients, ${ }^{15,16}$ but our patient was heavily sedated with propofol $(50 \mathrm{mg}$ propofol intravenously before retrobulbar block is a common practice in our unit and has been successfully administered without significant adverse events in more than 15,000 patients). This patient was young and a known drug abuser; hence, a second dose of $30 \mathrm{mg}$ propofol was slowly administered to keep him sedated for the procedure. He did not show features of deep sedation at any stage during propofol sedation. We cannot completely rule out propofol as the causative agent. Although propofol has been incriminated in inducing convulsions, ${ }^{17}$ it has also been used in the management of status epilepticus. ${ }^{18}$

Neurologic progression of the original disease might be considered, but negative results of subsequent neurologic investigations probably exclude this possibility. Because the patient had intrathecal 
chemotherapy and radiation causing retinitis, he might have had some adhesions causing disruption or abnormal flow of cerebrospinal fluid. This disruption perhaps led to slow tracking and progression of the local anesthetic to the brain (thus delayed clinical features) or even preferential tracking of cerebrospinal fluid flow so that it did not reach the brainstem because of significant uptake of the local anesthetic by other parts of the brain. In retrospect, a specific test looking at the cerebrospinal fluid dynamics might have proved very useful. We are not aware of any published study that suggests any change in the threshold for seizure in nicotine abusers.

Convulsions are reported after retrobulbar injection, but they are usually associated with other complex signs and symptoms of central nervous system toxicity. When the local anesthetic agent enters the central nervous system, it generally results in a complex phenomenon known as "brain stem anesthesia," quoted to vary from $0.3 \%$ to $0.8 \% .^{19,20}$ The exact incidence of this complication is not known because the features of these are reported as a single case or case series. The entry of the local anesthetic agent into the central nervous system in previously reported cases is supported by clinical features, but how it spreads is subject to different interpretations and hypothesis.

The symptoms of brainstem anesthesia include various combinations of agitation, confusion, unconsciousness, difficulty in breathing or apnea, cyanosis, numb throat, dysphagia, impaired hearing, hypertension, tachycardia, amaurosis in the contralateral eye, and shivering. ${ }^{19,20}$ Convulsions are known to occur presumably secondary to hypoxia and are usually associated with loss of consciousness, respiratory arrest, and cardiovascular collapse requiring artificial ventilation and vasopressor support. ${ }^{19}$ In most cases, symptoms appear within 2 minutes but may be delayed for a few minutes.

It is usually suggested that the local anesthetic agent unintentionally enters the optic nerve sheath and then tracks back into the subdural or subarachnoid space. The dura mater surrounds the optic nerve to the posterior aspect of the sclera. Drysdale ${ }^{21}$ showed in a cadaver that, when a needle is placed inside the optic nerve sheath and $3 \mathrm{~mL}$ contrast material is injected, the radiopaque dye is seen tracking along the nerve, chiasm, pons, and midbrain. The tracking can also be seen in reverse direction. This was proved in another study in which the contrast material was injected into the subarachnoid space, and it was noted to appear in the optic nerve sheath. ${ }^{22}$

Unintentional subarachnoid injection without brainstem anesthesia is the most plausible explana- tion in our case, which produced ipsilateral convulsions 9 minutes after the retrobulbar injection. Probably, the local anesthetic agent unintentionally injected inside the dural cuff surrounding the optic nerve spread to the optic chiasm and then to the right cerebral lobe producing local convulsions. The reason for convulsions suggested by Moorthy et al. ${ }^{23}$ is very convincing in our case. There is a selective blocking of inhibitory synapses because the excitatory synapses are more resistant to local anesthetic depression, leading to convulsions. ${ }^{24}$ The amygdaloid nucleus (amygdale) plays a central role. Amygdalae are complex subcortical structures closely associated with basal ganglia. They are connected with the reticular formation, hypothalamus, septal area, and olfactory areas of the forebrain. Rhythmic stimulation of amygdalae can produce convulsions. A further increase in the dose of local anesthetic agent probably caused inhibition of both inhibitory and facilitatory circuits on the right side of the brain, resulting in hemiparesis on the left side. The duration of action of $1 \%$ ropivacaine is comparable to $0.75 \%$ bupivacaine ${ }^{25}$; hence, it was expected to last longer. However, on the contrary, its duration was short lived. Muscle functions recovered faster than expected because ropivacaine probably did not reside long enough in the orbit to produce a longer duration block or entered into the cerebrospinal fluid or was metabolized, diluted, or redistributed. It is also envisaged that the local anesthetic agent did not reach midbrain and pons in sufficient quantity in our patient. Hence, there was no effect on cardiovascular or respiratory system, and the patient did not require cardiovascular support and needed only brief ventilatory support.

In retrospect, alternative relatively safer blocks, such as peribulbar or sub-Tenon's blocks, might have been a better choice in this patient. Although a peribulbar block is considered to be relatively safer than retrobulbar block, both sight and life-threatening complications have been reported..$^{20}$ The subTenon's block could have proved to be a safer alternative, but the technique is difficult to perform in younger patients because of toughness of the Tenon capsule. ${ }^{26}$ This block is also unsuitable for repeat procedures because the risk of complications increases if there is previous scarring. ${ }^{26}$

In conclusion, we present an unusual case of delayed ipsilateral convulsions followed by convulsions and paresis in the contralateral arm and leg after retrobulbar injection. Although these complications were self-limiting, they nevertheless required immediate attention. The exact cause or mechanism of the central spread of local anesthetic agent is not apparent. 


\section{References}

1. Leaming DV. Practice styles and preferences of ASCRS members-2003 survey. J Cataract Refract Surg 2004;30: 892-900.

2. Eke T, Thompson JR. The national survey of local anaesthesia for ocular surgery. II. Safety profiles of local anaesthesia techniques. Eye 1999;13:196-204.

3. Eke T, Thompson JR. Safety of local anaesthesia for cataract surgery: Why we should look again. Eye 2003;17:127-128.

4. Atkinson WS. Local anesthesia in ophthalmology. Trans Am Ophthalmol Soc 1934:32;399-451.

5. Hamilton RC. A discourse on the complications of retrobulbar and peribulbar blockade. Can J Ophthalmol 2000;35:363-372.

6. Hamilton RC. Complications of retrobulbar and peribulbar blocks. Reg Anesth 1990;15:106-107.

7. Troll GF. Regional ophthalmic anesthesia: Safe techniques and avoidance of complications. $J$ Clin Anesth 1995;7:163-172.

8. Rubin AP. Complications of local anaesthesia for ophthalmic surgery. Br J Anaesth 1995;75:93-96.

9. Kumar CM, Dodds C, Fanning GL. Ophthalmic Anaesthesia. The Netherlands: Sweats and Zeitlinger; 2002.

10. Ahn JC, Stanley JA. Subarachnoid injection as a complication of retrobulbar anesthesia. Am J Ophthalmol 1987; 103:225-230.

11. McLure HA, Rubin AP. Review of local anaesthetic agents. Minerva Anestesiol 2005;71:59-74.

12. Moorthy SS, Zaffer R, Rodriguez S, Ksiazek S, Yee RD. Apnea and seizures following retrobulbar local anesthetic injection. J Clin Anesth 2003;15:267-270.

13. Rozentsveig V, Yagev R, Wecksler N, Gurman G, Lifshitz T. Respiratory arrest and convulsions after peribulbar anesthesia. J Cataract Refract Surg 2001;27: 960-962.

14. Aldrete JA, Romo-Salas F, Arora S, Wilson R, Rutherford R. Reverse arterial blood flow as a pathway for central nervous system toxic responses following in- jection of local anesthetics. Anesth Analg 1978;57: 428-433.

15. Meyers EF, Ramirez RC, Boniuk I. Grand mal seizures after retrobulbar block. Arch Opthalmol 1978; 96:847.

16. Alsaadi TM, Marquez AV. Psychogenic nonepileptic seizures. Am Fam Physician 2005;72:849-856.

17. Finley GA, MacManus B, Sampson SE, Fernandez CV, Retallick R. Delayed seizures following sedation with propofol. Can J Anaesth 1993;40:863-865.

18. Brown LA, Levin GM. Role of propofol in refractory status epilepticus. Ann Pharmacother 1998;32:10531059.

19. Hamilton RC. Brain-stem anesthesia as a complication of regional anesthesia for ophthalmic surgery. Can J Ophthalmol 1992;27:323-325.

20. Nicoll JM, Acharya PA, Ahlen K, Baguneid S, Edge KR. Central nervous system complications after 6000 retrobulbar blocks. Anesth Analg 1987;66:1298-1302.

21. Drysdale DB. Experimental subdural retrobulbar injection of anesthetic. Ann Ophthalmol 1984;16:716718 .

22. Tenner MS, Trokel SL. Demonstration of the intraorbital portion of optic nerves by pneumoencephalography. Arch Ophthalmol 1968;79:572-573.

23. Moorthy SS, Zaffer R, Rodriguez S, Ksiazek S, Yee RD. Apnea and seizures following retrobulbar local anesthetic injection. J Clin Anesth 2003;15:267-270.

24. Covino BG. Clinical pharmacology of local anesthetic agents in neural blockade. In: Cousins MJ, Bridenbaugh PO, eds. Neural Blockade in Clinical Anesthesia and Pain Management. 2nd ed. Philadelphia: JB Lippincott Co; 1998:97-128.

25. Nicholson G, Sutton B, Hall GM. Comparison of $1 \%$ ropivacaine with $0.75 \%$ bupivacaine and $2 \%$ lidocaine for peribulbar anaesthesia. Br J Anaesth 2000; 84:89-91.

26. Kumar CM, Williamson S, Manickam B. A review of sub-Tenon's block: Current practice and recent development. Eur J Anaesthesiol 2005;22:567-577. 